\title{
APPROXIMATION OF SMOOTH FUNCTIONS
}

BY G. G. LORENTZ ${ }^{1}$

Communicated by R. P. Boas, January 13, 1960

Theorems of Jackson and S. Bernstein about the approximation of smooth functions are usually interpreted in the way that all functions with a prescribed degree of smoothness have a definite degree of approximation. They can be viewed in another way, which reveals their susceptibility to generalization.

Let $\omega(h)$ be an increasing continuous subadditive function defined for $h \geqq 0$ with $\omega(0)=0, A$ a compact metric space with infinitely many points. By $C_{1}^{\omega}$ we denote the set of all real valued functions $f$ on $A$ with $|f(x)| \leqq 1,\left|f(x)-f\left(x^{\prime}\right)\right| \leqq \omega(h), h=\rho\left(x, x^{\prime}\right)$. If $A$ is a $q$ dimensional cube, $p$ a natural number and $0<\alpha \leqq 1$, we denote by $C_{1}^{p+\alpha}$ the set of all functions on $A$ with continuous partial derivatives of orders not exceeding $p$ and bounded by 1 , and with the derivatives of order $p$ satisfying a Lipschitz condition of order $\alpha$ and with coefficient 1 . Let $G=\left\{g_{n}\right\}$ be a sequence of continuous functions on $A$. Then, with some norm, for example the uniform norm on $A$,

$$
E_{n}(f)=E_{n}^{\sigma}(f)=\inf \left\|f-\sum_{i=1}^{n} a_{i} g_{i}\right\|
$$

is the degree of approximation of $f$ by linear combinations of $g_{1}, \cdots, g_{n} ;$ and

$$
\varepsilon_{n}(W)=\sup _{f \in W} E_{n}(f)
$$

is the degree of approximation of a class $W$.

The theorems of Jackson and Bernstein state that for periodic $f \in C_{1}^{p+\alpha}$, and the trigonometric approximation, $E_{n}(f)$ has the exact order $n^{-(p+\alpha) / q}$; exceptions occur only if $f$ has a higher degree of smoothness. We regard this as a statement about a certain massivity of $C_{1}^{p+\alpha}$, which prevents better approximation by linear combinations of only $n$ functions. One can hope that an estimate of $\varepsilon_{n}\left(C_{1}^{p+\alpha}\right)$ from below can be given for an arbitrary system $G$, and that the trigonometric system is close to the best possible. That this is true, is shown by the following results:

TheOREM 1. Let $A$ be a compact metric space, and $\delta=\delta(n)$ the largest

1 This research was in part supported by the United States Air Force under Contract No. AF49(638)-619 monitored by the AFOSR of the Air Research and Development Command. 
number such that there exist $n$ points of $A$ with mutual distances $\geqq \delta$. Then for each $G$,

$$
\mathcal{E}_{n}\left(C_{1}^{\omega}\right) \geqq \frac{1}{2} \omega(\delta(n+1))
$$

This cannot be essentially improved, for there exists a $G$ with $\mathcal{E}_{n}\left(C_{1}^{\omega}\right) \leqq \omega\left(\delta_{1}\right)$, if $A$ can be covered by $n$ open balls of radius $\delta_{1}$ $=\delta_{1}(n)$.

Theorem 2. If $A$ is a $q$-dimensional cube, then for some constant $B$, and each $G$, in the uniform and the $L^{1}$ norm

$$
\mathcal{E}_{n}\left(C_{1}^{p+\alpha}\right) \geqq B n^{-(p+\alpha) / q}, \quad \quad p=0,1, \cdots ; 0<\alpha<1 .
$$

From these and similar theorems one can obtain by a method of condensation of singularities:

Theorem 3. If $A$ is as in Theorem $2, p=0,1, \cdots$ and $0<\alpha<1$, then there exists a constant $B$ such that for each system $G$ one can find $a$ function $f_{0} \in C_{1}^{p+\alpha}$ such that, in the uniform and the $L^{1}$ norm,

$$
E_{n}\left(f_{0}\right) \geqq B n^{-(p+\alpha) / q}
$$

for an infinite number of values of $n$.

Theorem 4. Let $A_{\rho}$ be the ellipse with focii $-1,+1$ and the sum of the half-axes $2 \rho$. For each $G$ and each sequence $\epsilon_{n} \rightarrow 0$ there exists a function $f_{0}(z)$, analytic inside $A_{\rho}$, with $\left|f_{0}(z)\right| \leqq 1$ such that the degree of approximation of $f_{0}$ on $(-1,+1)$ satisfies, in the $L^{1}$ norm,

$$
E_{n}\left(f_{0}\right) \geqq \epsilon_{n} \rho^{-n}
$$

for infinitely many $n$.

Similar and more general results were recently obtained by A. G. Vituškin [1]. However, his lower bounds (for $s=n+1, m=0$ in [1]) are of orders $(n \log n)^{-(p+\alpha) / 2 q}$ and $n^{-a n}, a>0$, for the problems of types (1), and (2), respectively.

\section{REFERENCE}

1. A. G. Vituškin, Best approximation of differentiable and analytic functions, Dokl. Akad. Nauk SSSR vol. 119 (1958) pp. 418-420.

SyracUSE UNIVERSITY 